\title{
ESTUDIO PRELIMINAR DE LA MACROFAUNA DEL SUELO EN ÁREAS DE VARILLALES Y CHAMIZALES DE LA AMAZONÍA PERUANA
}

\section{Sandra Tapia-Coral ${ }^{1}$, Beto Pashanasi ${ }^{2}$ y Dennis del Castillo ${ }^{3}$}

\section{RESUMEN}

La densidad (individuos $/ \mathrm{m}^{2}$ ) y biomasa ( $\mathrm{g}$ de peso fresco $/ \mathrm{m}^{2}$ ) de la macrofauna del suelo, fueron estudiadas en las áreas de varillales y chamizales de una parte de la Amazonía peruana. El estudio fue desarrollado en el Centro de Investigaciones de Jenaro Herrera (CIJH), $200 \mathrm{~km}$ sur oeste de la ciudad de Iquitos, en la margen derecha del río Ucayali, y en la Zona Reservada Allpahuayo-Mishana, situada en el km 29 de la carretera Iquitos-Nauta.

El método de muestreo utilizado fue recomendado por el Programa «Tropical Soil Biology and Fertility» (TSBF), y se realizó en los meses de marzo (época lluviosa) y setiembre (época de menos lluvias) del 2001. En cada sistema se colectaron 3 muestras, a un intervalo de 5 metros a lo largo de una línea recta cuyo origen y dirección fue escogido al azar, y la macrofauna fue separada en forma manual en monolitos de 25 $\mathrm{cm} \times 25 \mathrm{~cm} \times 30 \mathrm{~cm}$.

En los varillales del CIJH se encontró una densidad poblacional de 1611 a 2781 individuos $/ \mathrm{m}^{2}$ y una biomasa de 30.3 a $42.7 \mathrm{~g}$ peso fresco $/ \mathrm{m}^{2}$ en la época lluviosa y menos lluviosa, respectivamente. En la Zona Reservada de Allpahuayo-Mishana, la densidad fue de 10209 individuos $/ \mathrm{m}^{2}$ en la época lluviosa, y 768 individuos $/ \mathrm{m}^{2}$ en la época menos lluviosa. La biomasa tuvo un rango de 236.9 a $96.2 \mathrm{~g}$ de peso fresco $/ \mathrm{m}^{2}$ en las dos épocas de muestreo.

La densidad poblacional en los chamizales de Allpahuayo-Mishana fueron de 670 a 1179 individuos $/ \mathrm{m}^{2}$ y una biomasa de $31.5 \mathrm{a} 100.0 \mathrm{~g}$ de peso fresco $/ \mathrm{m}^{2}$ en la época lluviosa y menos lluviosa, respectivamente. En el CIJH se encontró una densidad de

1 Estudiante de Doctorado, INPA-Ecología, Manaus, AM, Brasil, sandra@inpa.gov.br

2 F. Zootecnia - UNAP, Yurimaguas-Perú

3 IIAP, Iquitos-Perú 
1728 a 4205 individuos $/ \mathrm{m}^{2}$ y la biomasa estuvo en un rango de 31.3 a $42.0 \mathrm{~g}$ de peso fresco $/ \mathrm{m}^{2}$, en las mismas épocas de muestreo.

Palabras claves: Macrofauna, Varillal, Chamizal, Densidad, Biomasa.

\section{SUMMARY}

Density (ind $/ \mathrm{m}^{2}$ ) and biomass ( $\mathrm{g}$ of fresh weight $/ \mathrm{m}^{2}$ ) of soil macrofauna were studied on the varillales and chamizales areas in a section of the Peruvian Amazon. The study was conducted on two sites: Jenaro Herrera Research Center (CIJH), located at $200 \mathrm{~km} \mathrm{SW}$ of the city of Iquitos on the right riverbank of the Ucayali river, and Allpahuyo-Mishana Reserved Zone, located on the $29 \mathrm{~km}$ of the Iquitos-Nauta highway. The soil macrofauna sampling was made according to the sampling method recommended by the "Tropical Soil Biology and Fertility" (TSBF) Program, during the months of March (rainy season) and September (less rainy) in 2001. For each system, three samples were taken along a line at a 5 meter interval. The origin and direction of the line were chosen at random, and the macrofauna was separated in monoliths of $25 \mathrm{~cm} \mathrm{X} 25 \mathrm{~cm} \mathrm{X} 30 \mathrm{~cm}$.

In the varillales of the CIHJ, population densities of 1611 and 2781 individuals $/ \mathrm{m}^{2}$, and a biomass of 30.3 and $42.7 \mathrm{~g}$ of humid weight $/ \mathrm{m}^{2}$, were found during the rainy and less rainy season, respectively. In the Allpahuayo-Mishana Reserved Area, density was 10209 individuals $/ \mathrm{m}^{2}$ during the rainy season and 768 individuals $/ \mathrm{m}^{2}$ during the less rainy season. Biomass was 236.9 and $96.2 \mathrm{~g}$ of humid weight $/ \mathrm{m}^{2}$ for the two sampling periods.

Population density in the chamizales of Allpahuayo-Mishana was 670 and 1179 individuals $/ \mathrm{m}^{2}$ and biomass was 31.5 and $100.0 \mathrm{~g}$ of humid weight $/ \mathrm{m}^{2}$, during the rainy and less rainy seasons, respectively. In the CIJH, population density was from 1782 to 4205 individuals $/ \mathrm{m}^{2}$ and biomass was 31.3 and $42.0 \mathrm{~g}$ of humid weight $/ \mathrm{m}^{2}$, during the same sampling periods.

Key words: Macrofauna, Varillal, Chamizal, Density, Biomass. 


\section{INTRODUCCIÓN}

La Amazonía peruana, con una superficie de aproximadamente $770000 \mathrm{~km}^{2}$, es un ecosistema muy complejo, dotado de diferentes tipos de vegetación. Algunos de estos tipos causan un extraño contraste con la exuberancia del bosque tropical y son comúnmente conocidos como varillales y chamizales en la Amazonía peruana (Encarnación, 1993), y campinaranas y campinas en la Amazonía brasileña (Anderson et al., 1975; Encarnación, 1993).

En estos tipos de vegetación, la macrofauna del suelo ejerce una función esencial sobre la estructura del suelo y el ciclo de nutrientes. La diversidad y la abundancia de algunos grupos de la fauna del suelo también pueden ser consideradas importantes indicadores de la fertilidad del suelo (Tian et al., 1997; Tapia-Coral et al., 1999). El suelo abriga un gran número de invertebrados, desde ácaros y colembolos hasta isopteros, formicidos y oligoquetos, que ocupan un gran espacio en densidad y biomasa en la Amazonía peruana y brasileña (Lavelle et al., 1992; Tapia-Coral et al., 1999; Barros, 1999). Esta fauna es extremadamente compleja, tanto estructural como funcionalmente, y es poca conocida en la Amazonía peruana.

La estructura y abundancia de las comunidades de la macrofauna del suelo son muy sensibles a las modificaciones de la cobertura vegetal del suelo (Lavelle et al., 1992). Por lo tanto, la interferencia humana en la Amazonía está causando cambios en los procesos que mantienen la sostenibilidad de sus ecosistemas (Barbosa y Fearnside, 1996). La deforestación es la amenaza más seria para la conservación de los recursos naturales de la Amazonía, siendo la agricultura migratoria la principal responsable de la deforestación alcanzando $69482 \mathrm{~km}^{2}$ hasta 1990 (INRENA, 1997). Esto representaba $9.20 \%$ del total cubierto por el bosque primario.

El objetivo del presente estudio fue realizar una evaluación preliminar de la macrofauna del suelo sobre las áreas de varillales y chamizales de áreas no inundables de la Amazonía peruana.

\section{METODOLOGÍA}

El trabajo fue realizado en el Centro de Investigaciones de Jenaro Herrera (CIJH) y en la Zona Reservada Allpahuayo-Mishana (ZRAM) (Figura 1). 


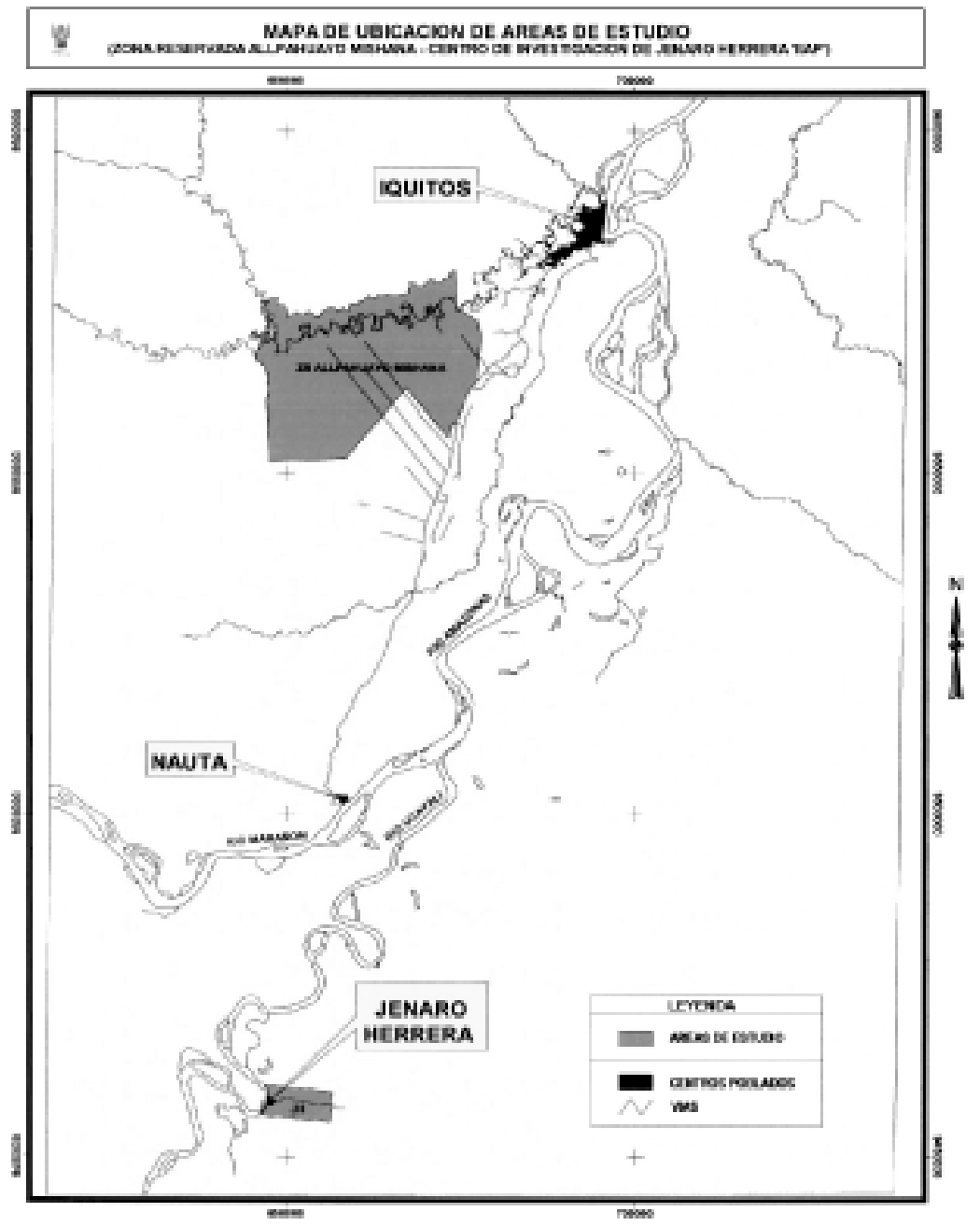

Figura 1. Mapa de las áreas de estudio: CIJH en Jenaro Herrera y la ZRAM en la Carretera Iquitos-Nauta. 
El CIJH, se encuentra situado a $200 \mathrm{~km}$ sur oeste de la ciudad de Iquitos, en la margen derecha del río Ucayali, departamento de Loreto, provincia de Requena, distrito de Villa Jenaro Herrera, en las coordenadas $73^{\circ} 40^{\prime}$ oeste y $4^{\circ} 54^{\prime}$ sur. Jenaro Herrera presenta una temperatura media mensual de $26.4^{\circ} \mathrm{C}$, y una precipitación media anual de 2,687 mm (Claussi et al., 1992).

Durante los muestreos en las áreas de varillales y chamizales del CIJH, los datos de precipitación y temperatura fueron registrados en la estación meteorológica del CIJH, encontrándose una precipitación de 410 y $195 \mathrm{~mm}$ para los meses de marzo y setiembre del año 2001, respectivamente (Figura 2).

La ZRAM se encuentra ubicada muy cerca de la ciudad de Iquitos (departamento de Loreto, provincia de Maynas, distrito de San Juan), y tiene una superficie de 57667.43 ha, con una precipitación anual entre 2,500 y 3,000 mm y una temperatura media anual mayor a $26^{\circ} \mathrm{C}$.

Durante los muestreos en la reserva, los datos de precipitación y temperatura, fueron tomados de la Estación Meteorológica del Instituto Nacional de Investigación Agropecuaria (INIA), situado cerca de los lugares de muestreo, verificándose una precipitación de $367.2 \mathrm{~mm}$ en el mes de marzo, y de $145.7 \mathrm{~mm}$ en el mes de setiembre (Figura 2). 


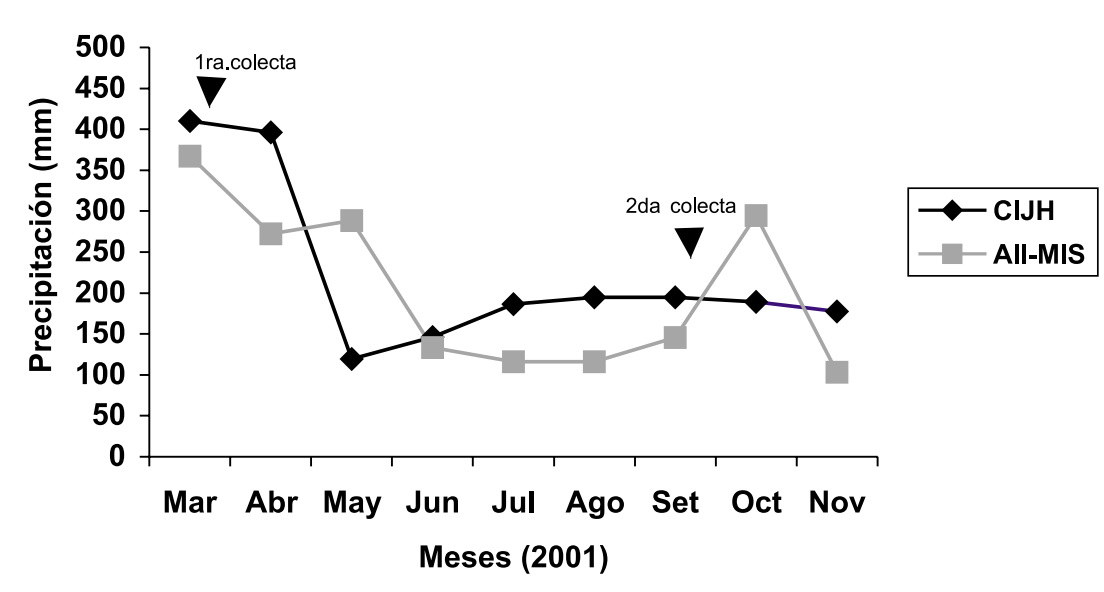

Figura 2. Precipitación ( $\mathrm{mm}$ ) mensual durante el período de estudio en el Centro de Investigaciones de Jenaro Herrera y en la Zona Reservada AllpahuayoMishana. Las flechas indican los meses de los muestreos (marzo y setiembre del 2001).

En el CIJH, los varillales se encuentran cercanos al distrito de Jenaro Herrera y siempre continuos a los chamizales ( $73^{\circ} 37^{\prime} 57^{\prime \prime}$ oeste y $4^{\circ} 54^{\prime} 125^{\prime \prime}$ sur). El suelo presenta una capa de humus negruzco hasta una profundidad de $15 \mathrm{~cm}$, seguida de la capa de arena blanca. La vegetación es muy densa, con árboles delgados y rectos, con troncos de más de $3 \mathrm{~cm}$ de DAP y hasta 10 - $20 \mathrm{~m}$ de alto; las especies más comunes son: "sacha punga", "aceite caspi", "ungurahui", "chambira", "cashapona" "remocaspi" y "chullachaqui" (Ver Anexo 1). Los líquenes, helechos, orquídeas y bromeliáceas también destacan entre las epífitas.

Los chamizales, se encuentran sobre suelos de arena blanca a partir de $5 \mathrm{~cm}$ de profundidad; los árboles tienen típicas formas enanas, con el tronco recto y delgado y es muy común encontrar a la palmera "aguajillo" y a los pungales (Ver Anexo 1). Al centro se observa una vegetación sobre montículos húmicos con helechos asociados a bromelias. Los chamizales siempre están circundados por los varillales (Encarnación, 1993). 
La ZRAM presenta cuatro grandes comunidades vegetales, adaptadas al relieve, a la naturaleza del suelo, a las condiciones de inundación y al drenaje: a) Relieve de terrazas ondulado-disectadas, se encuentran en los bosques altos sobre suelos arcillosos, relativamente ricos (pertenecientes a la "Formación Pebas"); b) Bosques sobre suelos areno-arcillosos, con diferentes gradientes de drenaje; c) Bosques esclerófilos sobre arena blanca, con capas profundas y capas superficiales, llamados "varillales y chamizales", y d) Una formación vegetal que se ubica en las terrazas adyacentes y contiguas al río Nanay, sujeta a los flujos de inundación estacional de las aguas negras (INRENA et al., 2000).

Las colectas de macrofauna, se realizaron en los bosques esclerófilos sobre arena blanca identificados como varillal alto húmedo y chamizal húmedo (03⒌'23'" sur, $73^{\circ} 24^{\prime} 47^{\prime \prime}$ oeste). La riqueza taxonómica vegetal se muestra en el Anexo 3. Los varillales se encuentran continuos a los chamizales, al igual que en el CIJH.

La macrofauna del suelo fue muestreada, en el mes de marzo (época lluviosa) y en setiembre (época de menos lluvias) del 2001, utilizándose el método recomendado por el Programa "Tropical Soil Biology and Fertility" (TSBF/IUBS/UNESCO).

En las áreas de varillales y chamizales de Jenaro Herrera y Allpahuayo-Mishana se colectaron 3 muestras de monolitos de suelo $(25 \mathrm{~cm}$ x $25 \mathrm{~cm} \times 30 \mathrm{~cm}$ de profundidad), en cada una de las áreas, a lo largo de un transecto de línea recta de $20 \mathrm{~m}$, cuyo origen y dirección fue escogido al azar, a un intervalo de $5 \mathrm{~m}$ cada uno. Cada monolito era dividido en cuatro estratos sucesivos (hojarasca; 0-10;10-20;20-30 cm de profundidad) (Pashanasi, 2001). En la época de mayor precipitación (marzo) en algunos casos sólo fue posible muestrear hasta una profundidad de $20 \mathrm{~cm}$, debido a la abundante humedad en los monolitos muestreados, lo que imposibilitó los muestreos en todos los horizontes.

Para la colecta de los invertebrados del suelo se utilizó el control visual, revisando minuciosamente los extractos del suelo y se procedió a colectar manualmente con ayuda de pinzas entomológicas en el mismo campo. La macrofauna existente fue conservada en alcohol al $70 \%$ y las lombrices en formol al $4 \%$. En el laboratorio, la macrofauna fue identificada a nivel de orden, contada y pesada. La densidad fue medida en individuos $/ \mathrm{m}^{2}$ y la biomasa en gramos de peso fresco $/ \mathrm{m}^{2}$. 


\section{RESULTADOS}

\subsection{Macrofauna del suelo en áreas de varillales del Centro de Investigación Jenaro Herrera y Allpahuayo-Mishana}

En el muestreo efectuado en la época lluviosa (marzo), en los varillales del CIJH, fue encontrada una diversidad de 15 unidades taxonómicas, con una densidad poblacional de 2,781 individuos $/ \mathrm{m}^{2}$ (Anexo 3), de los cuales el 38.7\% fueron Formicidae y el $35.1 \%$ Isoptera; con una biomasa de $30.3 \mathrm{~g}$ de peso húmedo $/ \mathrm{m}^{2}$ (Anexo 3). La mayor biomasa fueron las larvas de Coleóptera (44.3\%), seguido de los Isoptera (24.1\%) (Figura 3). En la segunda colecta (época de menos lluvias), se encontró una diversidad de 12 unidades taxonómicas, con una densidad poblacional de 1611 individuos $/ \mathrm{m}^{2}$ (Cuadro 3), con dominancia de Formicidae (60\%), y una biomasa de $42.7 \mathrm{~g}$ de peso húmedo $/ \mathrm{m}^{2}$ (Cuadro 3). La biomasa de las larvas de Coleóptera alcanzó el 74.1\% (Figura 3).

E1 92.5\% del total de la población esta distribuido en las capa de hojarasca de 0-10 $\mathrm{cm}$ de profundidad en la época lluviosa y el $57.3 \%$ en la capa de $0-10 \mathrm{~cm}$, en la época de menos lluvia (Figura 4).

Los Varillales de la ZRAM tuvieron 21 unidades taxonómicas en la época lluviosa, con una densidad poblacional de 10209 individuos $/ \mathrm{m}^{2}$ (Anexo 3), dominados por el grupo de Isoptera con el $65 \%$. Esta abundancia de Isoptera, probablemente se debe a la presencia de una colonia en uno de los monolitos. La biomasa fue de $236.9 \mathrm{~g}$ de peso fresco $/ \mathrm{m}^{2}$ (Anexo 3), de los cuales el $62.4 \%$ lo conforman las Oligochaeta, seguido de los Isoptera con un $20.2 \%$ (Figura 3).

En el muestreo efectuado en la época de menos lluvias, en los varillales de AllpahuayoMishana, se encontró 15 grupos taxonómicos con una densidad poblacional de 768 individuos $/ \mathrm{m}^{2}$ (Anexo 3), dominados por los Formicidae (24.3\%) y los Coleoptera (20.8 \%). La biomasa de $96.2 \mathrm{~g}$ de peso fresco $/ \mathrm{m}^{2}$ (Anexo 3) es dominada por las larvas de Coleóptera (67\%) (Figura 3). El 73.1\% de la población de individuos se encontró en la capa de 0-10 cm y el 25.8 en la hojarasca, durante las colectas en la época lluviosa, y el $75 \%$ de la población en la capa de $0-10 \mathrm{~cm}$ en la época de menos lluvias (Figura 4). 


\section{Jenaro Herrera}

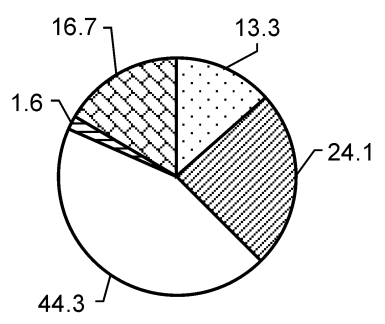

$30.3 \mathrm{~g}$

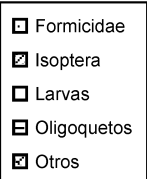

Otros

\section{Allpahuayo-Mishana}

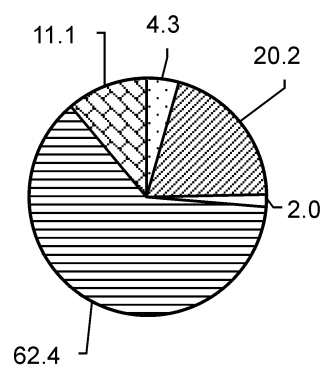

$236.9 \mathrm{~g}$

\begin{tabular}{|l|}
\hline$\square$ Formicidae \\
$\square$ Isoptera \\
$\square$ Larvas \\
$\square$ Oligoquetos \\
$\square$ Otros \\
\hline
\end{tabular}

a) Epoca lluviosa

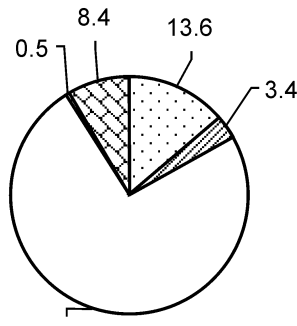

74.1

$42.7 \mathrm{~g}$

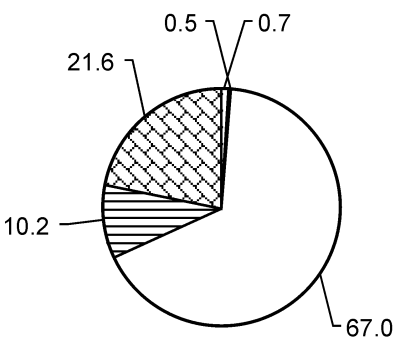

$96.2 \mathrm{~g}$

b) Epoca de menos lluvias

Figura 3. Biomasa (\%) de la macrofauna de suelo en áreas de Varillales del CI Jenaro Herrera y de la Zona Reservada Allpahuayo-Mishana, en la época lluviosa (a) y en época de menos lluvias (b). 


\section{Jenaro Herrera}
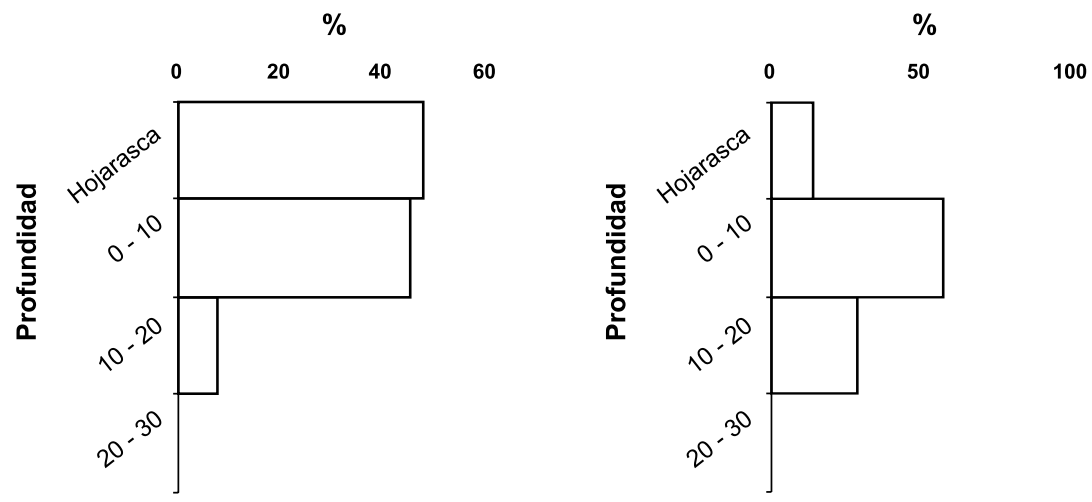

\section{Allpahuayo-Mishana}
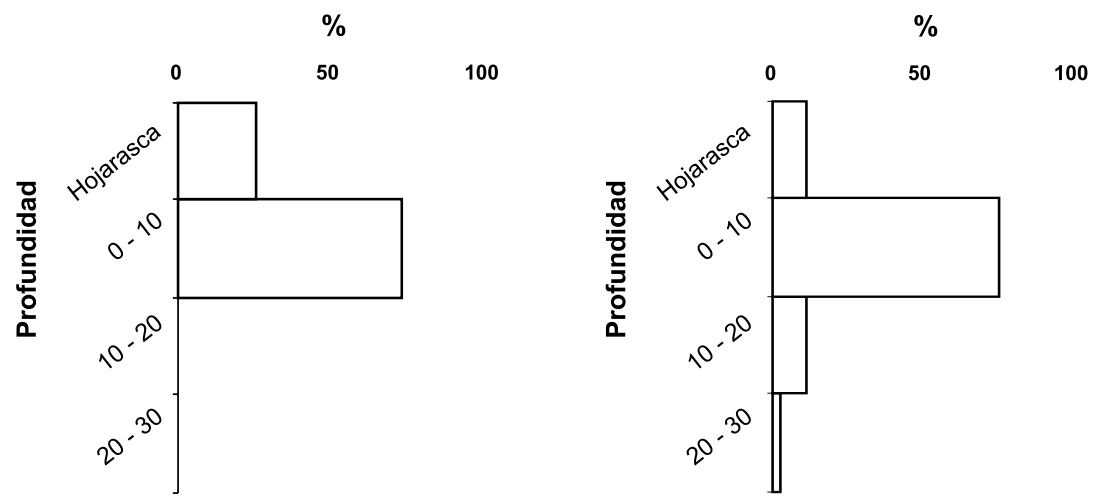
a) Época lluviosa
b) Época de menos lluvias

Figura 4. Distribución vertical de la macrofauna de suelo en los Varillales del CI Jenaro Herrera y la Zona Reservada Allpahuayo-Mishana, en la época lluviosa (a) y época de menos lluvias (b). 


\subsection{Macrofauna del suelo en áreas de chamizales del Centro de Investigación Jenaro Herrera y de la Zona Reservada Allpahuayo-Mishana}

En los chamizales del CIJH, durante la época lluviosa, sólo fue posible muestrear hasta la capa de 0-10 cm, ya que las demás capas estaban saturadas de agua. Se encontró una diversidad de 19 grupos taxonómicos, con una densidad poblacional de 4205 individuos $/ \mathrm{m}^{2}$ (Anexo 3), y donde los Formicidae conformaron el 71\%. La biomasa fue de $31.3 \mathrm{~g}$ de peso húmedo $/ \mathrm{m}^{2}$ (Anexo 3), de los cuales el $35.8 \%$ estuvo conformado por los Formicidae, seguido de los Oligochaeta (25.5\%) (Figura 5).

En la época de menos lluvias, los grupos taxonómicos fueron los más bajos (10) de todas las áreas colectadas, con una densidad poblacional de 1728 individuos $/ \mathrm{m}^{2}$ (Anexo 3), donde el grupo de los Isoptera conforman el $43 \%$, seguido por los Formicidae (35.5\%). La biomasa fue de $42.0 \mathrm{~g}$ de peso húmedo $/ \mathrm{m}^{2}$ (Anexo 3), donde las 2/3 partes de la biomasa lo conforman las larvas de Coleóptera (Figura 5).

En la Figura 5, se observa la distribución vertical de los componentes de la macrofauna, en donde se encontró que $57.3 \%$ de la población esta en la hojarasca y $42.7 \%$ en la capa de $0-10 \mathrm{~cm}$, en la época lluviosa. En la capa de $0-10 \mathrm{~cm}$ se encuentra el $75.3 \%$ de los individuos en la época de menos lluvias (Figura 6).

En la época lluviosa de los chamizales de la ZRAM se encontró una diversidad de 15 unidades taxonómicas, con una densidad poblacional de 670 individuos $/ \mathrm{m}^{2}$ (Anexo 3), donde el $23.1 \%$ esta formado por los Formicidae, seguido de los Isoptera (20.8\%). La biomasa fue de $100 \mathrm{~g}$ de peso húmedo $/ \mathrm{m}^{2}$ (Anexo 3), con dominancia de los Oligochaeta en $70.1 \%$ (Figura 5).

Los chamizales de Allpahuayo-Mishana, en la época de menos lluvias, sólo tuvieron 12 grupos taxonómicos, con una densidad poblacional de 1179 individuos $/ \mathrm{m}^{2}$ (Anexo 3), dominados por el grupo de los Formicidae en un $47.5 \%$. La biomasa fue de $31.5 \mathrm{~g}$ de peso húmedo $/ \mathrm{m}^{2}$ (Anexo 3), dominado por las larvas en $60 \%$ (Figura 5).

En la distribución vertical, se encontró que el $71.1 \%$ de los individuos se encuentran en la hojarasca, el $28.9 \%$ en la capa de $0-10 \mathrm{~cm}$, durante la época lluviosa, pasando lo contrario en la época de menos lluvias con un $68.8 \%$ en la capa de $0-10 \mathrm{~cm}$ (Figura 6). 


\section{Jenaro Herrera}

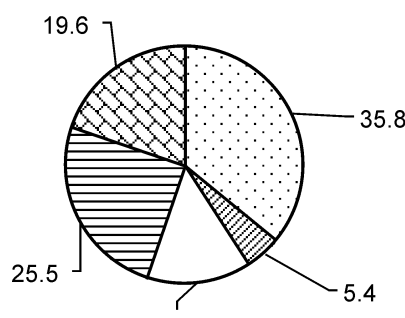

13.7

$31.3 \mathrm{~g}$

$$
\begin{aligned}
& \square \text { Formicidae } \\
& \square \text { Isoptera } \\
& \square \text { Larvas } \\
& \text { 日 Oligoquetos } \\
& \square \text { Otros }
\end{aligned}
$$

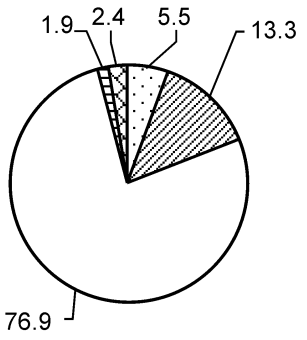

$42.0 \mathrm{~g}$

\section{Allpahuayo-Mishana}

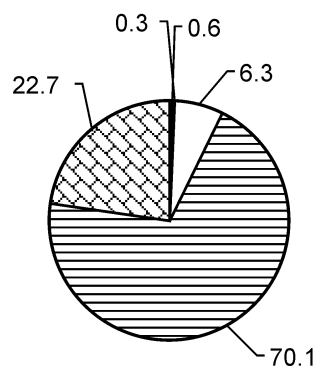

$100 \mathrm{~g}$

$$
\begin{aligned}
& \square \text { Formicidae } \\
& \square \text { Isoptera } \\
& \square \text { Larvas } \\
& \square \text { Oligoquetos } \\
& \square \text { Otros }
\end{aligned}
$$

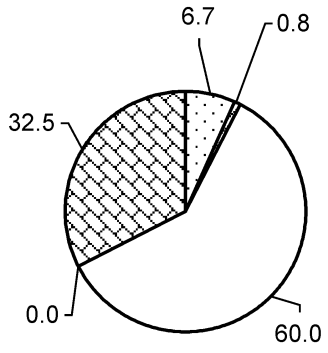

$31.5 \mathrm{~g}$

a) Epoca lluviosa

b) Epoca de menos lluvias

Figura 5. Biomasa de la macrofauna del suelo (\%) en áreas de chamizales en el CI Jenaro Herrera y en la Zona Reservada Allpahuayo-Mishana, en la época lluviosa (a) y época de menos lluvias (b). 


\section{Jenaro Herrera}
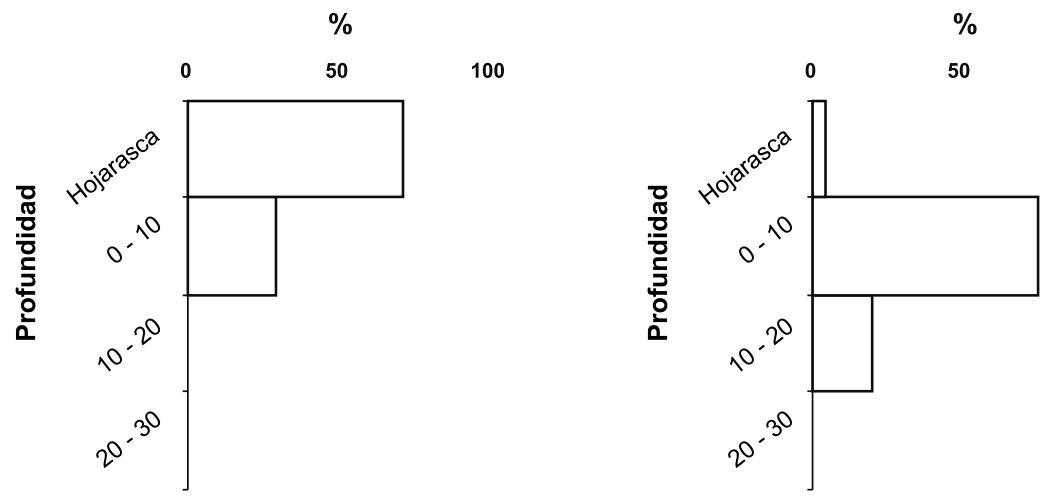

100

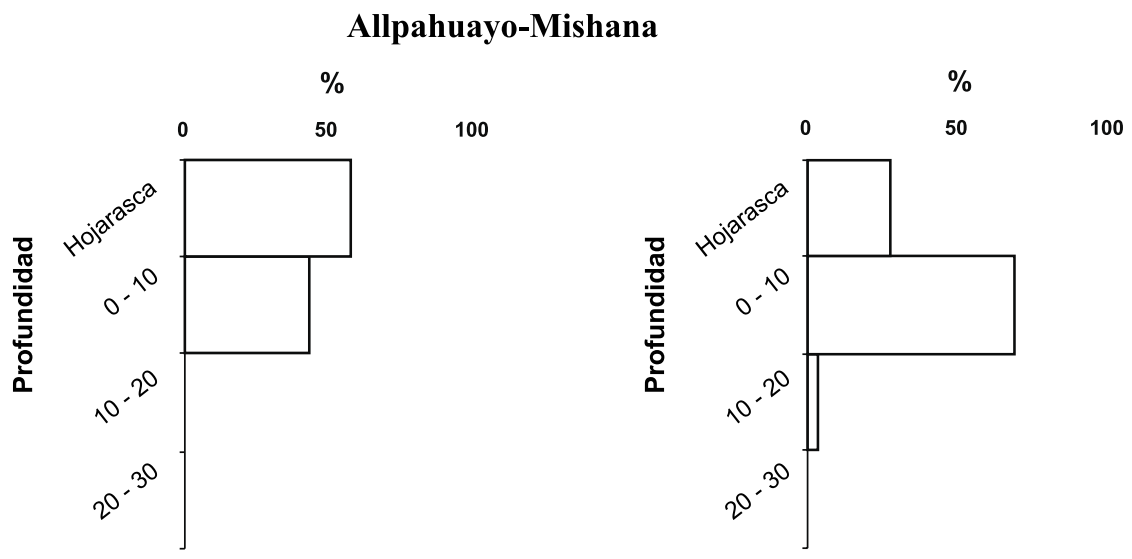

a) Época lluviosa

b) Época de menos lluvias

Figura 6. Distribución vertical de los componentes de la macrofauna de suelo en las áreas de chamizales en el CI Jenaro Herrera y en la Zona Reservada Allpahuayo-Mishana, en época lluviosa (a) y época de menos lluvias (b). 


\section{DISCUSIÓN}

La densidad y biomasa de la macrofauna en los sistemas de varillales y chamizales fueron bajas, en comparación a otros trabajos realizados en sistemas similares en la Amazonía brasileña (Luizão, 1995; Oliveira, 1996). La composición florística en estas áreas puede afectar la diversidad y densidad de la macrofauna del suelo; así mismo, puede ser influenciada por las propiedades físicas y químicas del suelo.

La densidad poblacional de individuos $/ \mathrm{m}^{2}$ fue mayor en los varillales de la ZRAM en la época lluviosa, en comparación a los varillales del CIJH (Anexo 3); sin embargo, en la segunda colecta fue lo contrario, es decir, una mayor densidad en los varillales del CIJH que en los varillales de la ZRAM (Anexo 3). La alta densidad en los varillales de la ZRAM en la primera colecta, se debe a que los dos principales componentes, Formicidae e Isoptera, conformaron el $92.6 \%$ del total de la población; y en los varillales del CIJH, el 73.8\%, respectivamente. Los Formicidae e Isoptera en la segunda colecta en los varillales de la ZRAM conforman solo el 33.3\%; pero en el varillal del CIJH la densidad de estos componentes es similar a la primera colecta $(72.5 \%)$. Los varillales de ambos lugares tienen un alto contenido de humus en la capa superior (aproximadamente 15 a $20 \mathrm{~cm}$ ), lo que favorecería la presencia de la fauna, y mantendría las condiciones de humedad más apropiadas para su actividad (Luizão, 1995).

La menor densidad de individuos $/ \mathrm{m}^{2}$ encontrada en las áreas de chamizales de la ZRAM (Anexo 3) puede deberse a la pobreza de la vegetación que no es uniforme (Anderson et al., 1975); esto es importante porque la vegetación favorece la creación de microhabitats que actúan directamente en la diversidad y distribución de la comunidad edáfica de invertebrados del suelo (Oliveira, 1996). Así mismo, el área se encuentra muy próxima a la carretera Iquitos-Nauta, donde hay un tránsito continuo de personas foráneas que estarían modificando la cobertura vegetal.

El grupo de los Formicidae, fueron los más abundantes en todas las áreas de muestreo, a excepción del chamizal de la ZRAM (en la época lluviosa) y en el varillal de la ZRAM, en la época de menos lluvias (Anexo 3), similar al trabajo efectuado en las áreas de varillales y chamizales de la Amazonía brasileña (Harada y Bandeira, 1994), donde hay dominancia del grupo de los Formicidae. En la Amazonía brasileña, los Formicidae constituyen los grupos de mayor densidad de la fauna del suelo (Fittkau y Klinge 1973; Bandeira y Harada, 1991), y junto con los Isoptera son considerados importantes descomponedores de la hojarasca del suelo de áreas no inundables (Luizão, 1995). En este estudio los Isoptera constituyen el segundo grupo de importancia en la 
época lluviosa en los varillales de ambos lugares, reduciéndose en la época de menos lluvia en los chamizales.

Los Oligochaeta tienen una importante biomasa en los suelos de la Amazonía peruana (Lavelle y Pashanasi, 1989), y en la Amazonía brasileña (Barros, 1999). Durante el presente estudio, los Oligochaeta presentaron una baja densidad y biomasa en las áreas muestreadas, principalmente porque la mayoría de las lombrices encontradas eran de la familia Nermitidae (lombrices muy pequeñas); sin embargo, en el chamizal del CIJH fueron encontrados en una densidad de 368 a 208 individuos $/ \mathrm{m}^{2}$ en las dos épocas de muestreo. (Anexo 3).

En áreas de chamizales de la Amazonía brasileña (Carretera Manaus-Boa Vista), Luizão (1995), verificó que Diplópoda e Isópoda tienen una alta densidad y biomasa en los suelos. Durante el presente estudio los Diplopodos tuvieron una población intermedia en la época lluviosa, de ambos lugares muestreados (Anexo 3), y una menor densidad en la época de menos lluvias; esto puede deberse a que los Diplopoda son muy sensibles a los cambios bruscos de temperatura y humedad (Adis et al., 1996).

Las larvas encontradas durante las dos épocas de muestreo en ambos lugares, tuvieron una alta biomasa (Figura 3 y 5), con predominancia de larvas de la familia Escarabidae (Coleoptera), de gran peso y tamaño, influyendo fuertemente en la biomasa total.

La distribución vertical de los componentes de la macrofauna del suelo estuvo concentrada en su gran mayoría en el horizonte de 0-10 $\mathrm{cm}$ de profundidad, en los varillales de ambos lugares y durante las dos épocas de colecta: lluviosa y menos lluviosa (Figura 4 y 6). Sin embargo, en los chamizales la mayor concentración estuvo en la hojarasca, y la capa de $0-10 \mathrm{~cm}$ de profundidad. En ambas capas es posible encontrar una humedad que estaría favoreciendo a la actividad de la macrofauna del suelo.

Es necesario realizar más investigaciones sobre la macrofauna de suelo, sobre todo a nivel de especie para los grupos mayoritarios: Formicidae por su alta densidad y Oligogochaeta por su biomasa, en estas áreas de estudio. 


\section{AGRADECIMIENTOS}

Los autores desean expresar su agradecimiento al Instituto de Investigaciones de la Amazonía Peruana (IIAP), al personal del Centro de Investigaciones de Jenaro Herrera (CIJH) y al Programa de Biodiversidad (PBIO), quienes proporcionaron apoyo y facilidades logísticas de campo, durante el período de muestreos en el CIJH y en la Zona Reservada Allpahuayo-Mishana. Del mismo modo, nuestro agradecimiento a la Dirección General de Áreas Naturales Protegidas del INRENA, por la autorización de investigación en la Zona Reservada Allpahuayo-Mishana. Un agradecimiento especial a nuestros asistentes de campo en el CIJH: Carlos Shapiama y Hugo Vásquez $\mathrm{y}$, a los guardaparques de la ZRAM Elías Aquituari y Juan Icomena. Igualmente agradecemos a Silvia del Águila y Joel Vásquez, por el apoyo en algunas colectas e identificación de la macrofauna. A Erasmo Otárola y Joel Vásquez por sus comentarios de la versión de este manuscrito.

\section{BIBLIOGRAFIA}

ADIS, J.; GOLOVATCH, S.I.; HAMANN, S. 1996. Survival strategy of the terricolous millipede Cutervodesmus adisi Golovatch (Fuhrmannodesmidae, Polydesmida) in a blackwater inundation forest of Central Amazonia (Brazil) in response to the flood pulse. Acta Myriapodologica, Mém. Mus. Hist. Nat. 169:523-532.

ANDERSON, A.B.; PRANCE, G.T.; ALBUQUERQUE, B.W.P. DE. 1975. Estudos sobre a Vegetacao das Campinas Amazônicas. III. A Vegetacao lenhosa da campina da Reserva Biológica INPA -SUFRAMA (Manaus-Caracaraí, km 62). Acta Amazônica, 5(3):225-246.

BANDEIRA, A.G.; HARADA, A.Y. 1991. Cupins e Formigas na Amazônia. In: Val, A.L.; Figliuolo, R.; Feldberg, E. (Eds.). Bases Científicas para Estratégias de Preservação e Desenvolvimento da Amazônia: Fatos e Perspectivas. Vol.1., Instituto Nacional de Pesquisas da Amazônia, Manaus, pp: 387-395.

BARBOSA, R.I.; FEARNSIDE, P.M. 1996. Carbon and nutrient flows in an Amazonian forest: fine litter production and composition at Apiaú, Roraima, Brazil. Tropical Ecology, 37(1):115-125. 
BARROS, E. 1999. Effet de la Macrofaune Sur la Structure et les Processus Physiques $d u$ Sol de Paturages Degrades D'Amazonie. Thèse de Doctorat de L’Université Paris 6. France. 127 pp.

BARROS, E.; NEVES, A.; FERNANDES, E.C.M.; WANDELLI, E.; LAVELLE, P. 2000. Soil macrofauna community of Amazonian Agroforestry Systems. Agroforestry Systems (no prelo).

CLAUSSI, A.; MARMILLOD, D. ; BLASER, J. 1992. Descripción silvicultural de las plantaciones forestales de Jenaro Herrera. Instituto de Investigaciones de la Amazonía Peruana (IIAP). Centro de Investigaciones de Jenaro Herrera. Iquitos, Perú. 225 p.

ENCARNACIÓN, F. 1993. El Bosque y las Formaciones Vegetales en la Llanura Amazónica del Perú. Alma Máter, 6: 95 -114.

FITTKAU, E.J.; KLINGE, H. 1973. On biomass and trophic structure of the Central Amazonian rain forest. Biotropica, 5(1):2-14.

HARADA, A.Y.; BANDEIRA, A.G. 1994. Estratificacao e Densidade de Invertebrados em Solo Arenoso sob Floresta Primária e Plantíos Arbóreos na Amazônia Central durante a Estacao Seca. Acta Amazonica, 24 (1/2):103118.

INRENA (Instituto Nacional de Recursos Naturales), 1997. Compendio Estadístico de la Actividad Forestal del Perú. Proyecto Monitoreo de la Deforestación en la Amazonía Peruana, DGFF. Lima. Perú. 77 p.

INRENA; IIAP; CTAR-L. 2000. Informe Final del "Estudio de Categorización y Delimitación de la Zona Reservada Allpahuayo-Mishana”. 107 p. (Documento técnico no publicado).

INPE. Brasil. 1998. Amazonia: Deforestation 1995-1997. Instituto Nacional de Pesquisas Espaciais (INPE). São José dos Campos, S.P. Brasil. Documento consultado via Internet.

LAVELLE, P.; PASHANASI, B. 1989. Soil macrofauna and land management in Peruvian Amazonia (Yurimaguas, Loreto). Pedobiologia, 33:283-291. 
LUIZ̃̃O, F. J. 1995. Ecological Studies in Contrasting Forest Types in Central Amazonia. Ph. D. Thesis. University of Stirling, Stirling, UK. 288 p.

OLIVEIRA, E.P. 1996. Estudo dos Invertebrados Terrestres e Distribuicao Vertical en diferentes Ecossistemas da Amazônia Central. XIII Congresso LatinoAmericano de Ciência do Solo, 1996. CD ROM, artigo 03-053 (5 pág) Software Gráfico Ltda. Campinas, SP.

PIRES, J.M.; PRANCE, G.T. 1985. The vegetation types of the Brazilian Amazon. In: Prance, G.T. and Lovejoy, T.E. (Eds. ). Key Environments: Amazonia, pp. 109-145.

RUOKOLAINEN, K.; TUOMISTO, H. 1993. La Vegetación de terrenos no inundables (tierra firme) en la selva baja de la Amazonía Peruana. In: R. Kalliola \& W. Danjoy (Eds). Amazonía Peruana. Vegetación húmeda tropical en el llano subandino. PAUT y ONERN, Jyvaskyla. pp. 139-153.

SWIFT, M.J., HEAL, O.W.; ANDERSON, J.M. 1979. Decomposition in terrestrial Ecosystems. Studies in Ecology 5. University of California Press, Berkeley.

TAPIA-CORAL, S.C. 1998. Macrofauna da Liteira em Sistemas Agroflorestais Implantados em Áreas de Pastagens Abandonadas na Amazônia central. Dissertação de Mestrado. INPA/FUA. 98 p.

TAPIA-CORAL, S.C.; LUIZÃO, F.; WANDELLI, E. 1999. Macrofauna da liteira em sistemas agroflorestais sobre pastagens abandonadas na Amazônia central. Acta Amazônica, 29 (3): 477-495.

TIAN, G.; KANG, B.T.; BRUSSARD, L. 1997. Effect of mulch quality on earthworm activity and nutrient supply in the humid tropics. Soil Biol. Biochem., 29:369373.

VOLHLAND, K.; SCHROTH, G. 1999. Distribution patterns of the litter macrofauna in agroforestry and monoculture plantations in Central Amazonia as affected by plant species and management. Applied Soil Ecology, (13): 57-68. 


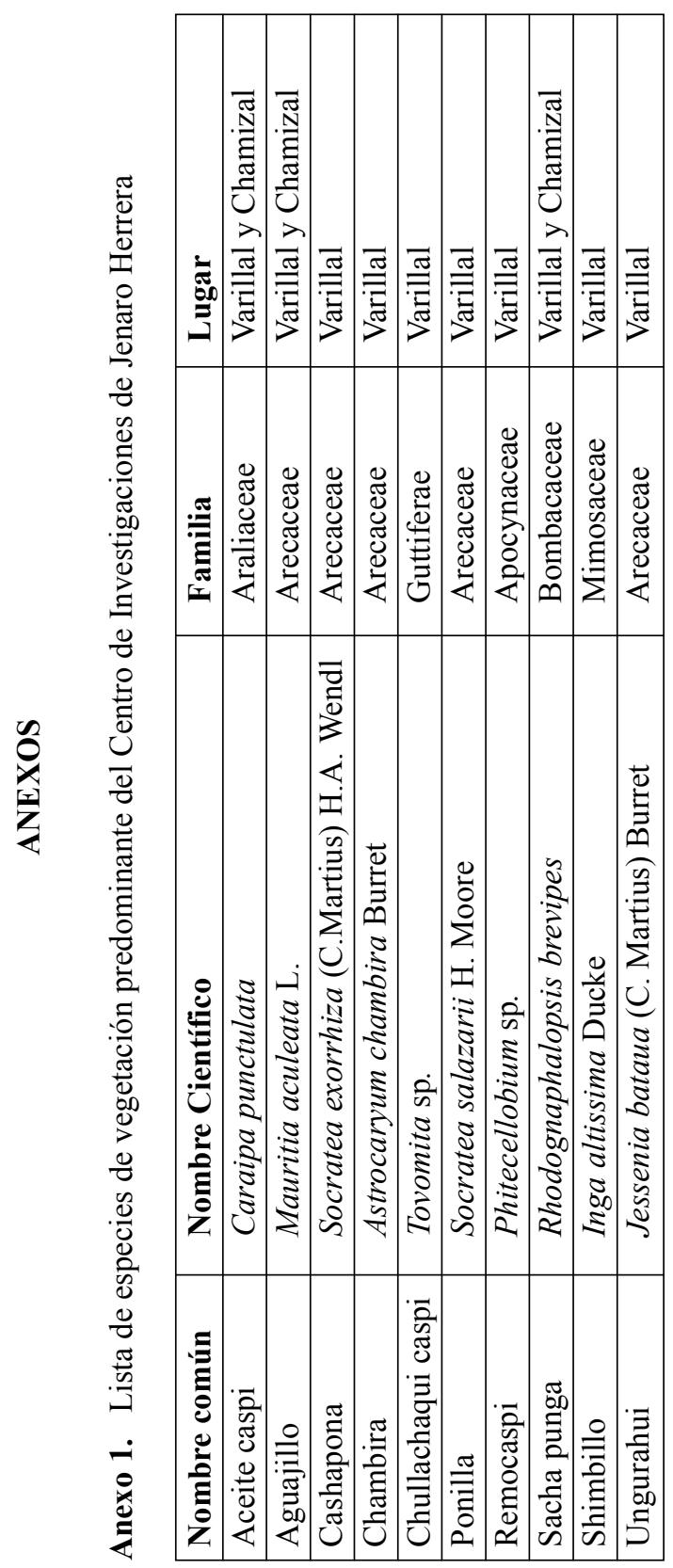




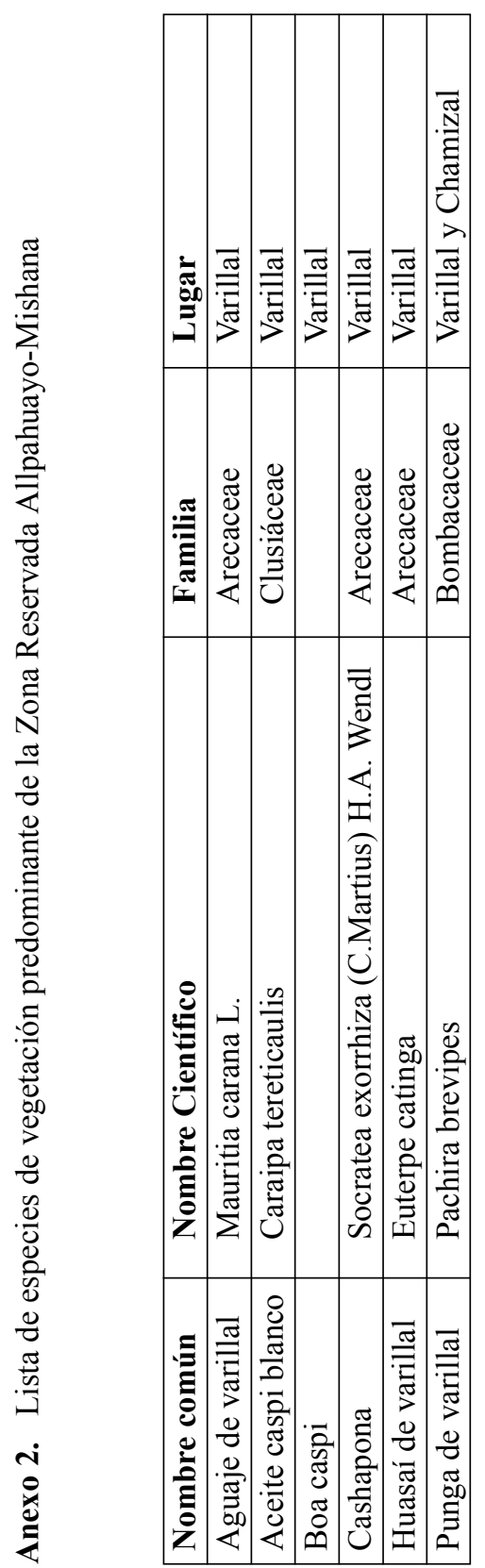


$\frac{0}{2}$

తี

苂

담

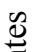

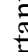

을

:

อ

.

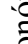

產

帘

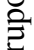

की

으 $\frac{0}{8}$

웜

สี

कo

उ

틍

응

入

สํํㄱ

\&

竞 $\frac{\dot{0}}{\tilde{D}}$

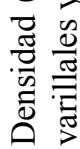

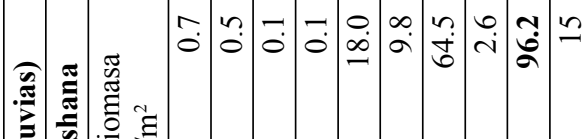

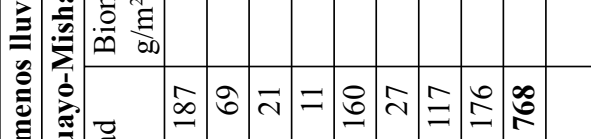

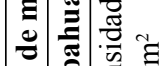

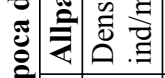

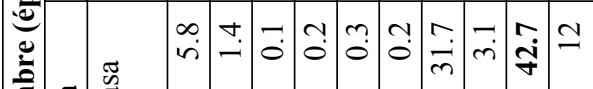

छี

包

苞

ن 巳

ปั่

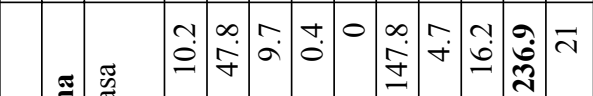

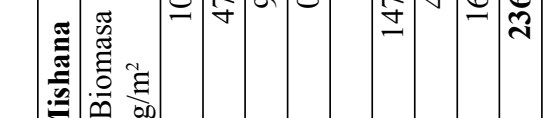

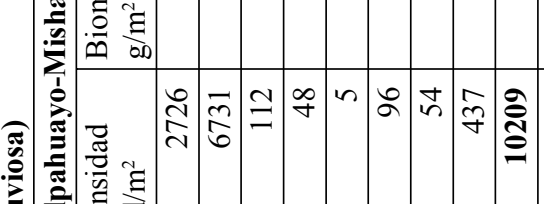

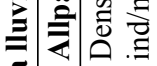

窇

ง

¿

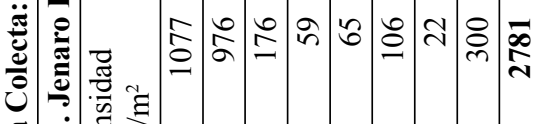

ت્త

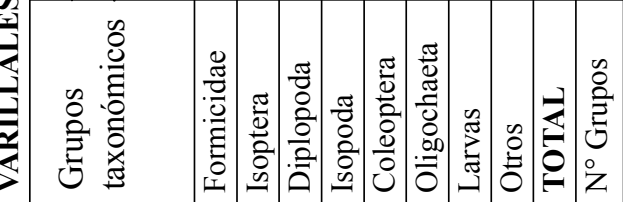




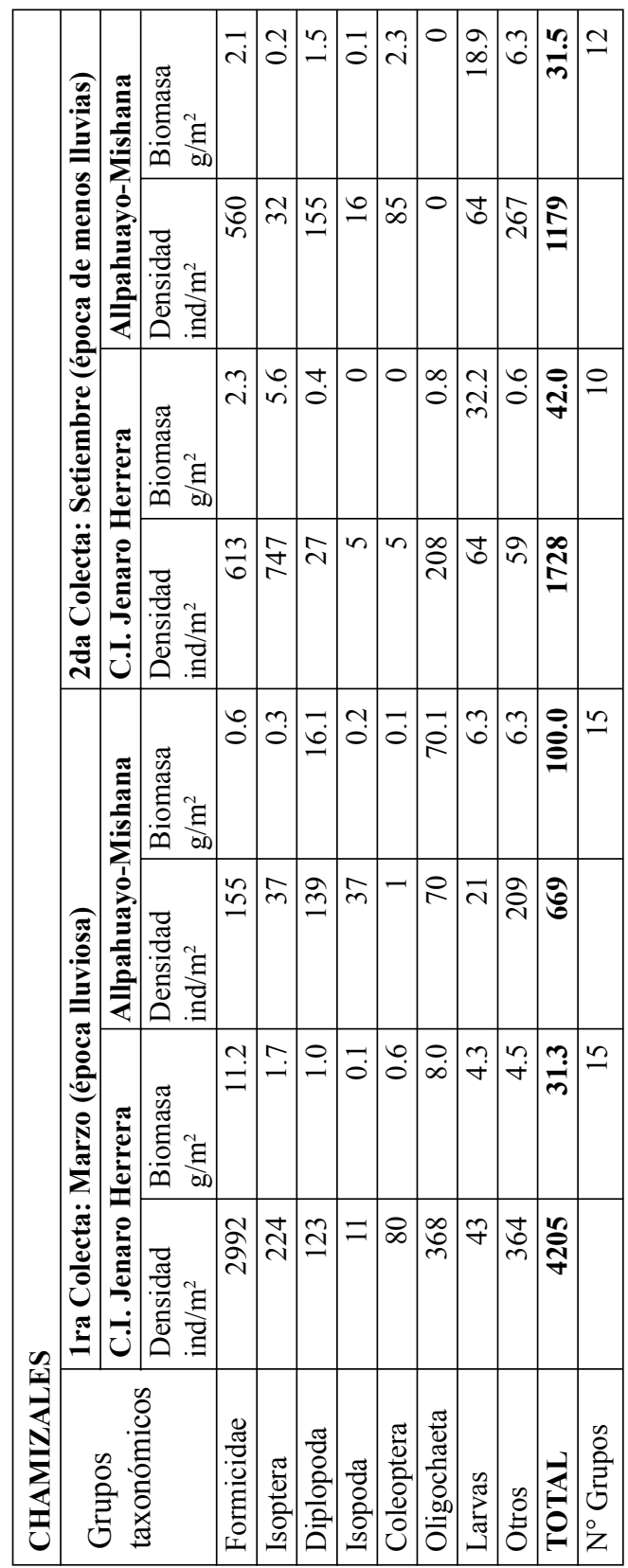

